\title{
Projection Reconstruction MR Imaging using FOCUSS
}

\author{
S. Tak ${ }^{1}$, Y. Han ${ }^{2}$, H. W. Park ${ }^{2}$, and J. C. Ye'
}

${ }^{1}$ BioSystems, Korea Advanced Institute of Science \& Technology, Yuseong-gu, Daejon, Korea, Republic of, ${ }^{2}$ Korea Advanced Institute of Science \& Technology

Introduction : The acquisition time of the Projection Reconstruction (PR) MR can be reduced by using angular under-sampling. However, under-sampled PR can result in aliasing artifacts. The main goal of this paper is to develop a novel reconstruction algorithm free of angular aliasing. We show that a sparse reconstruction algorithm called Focal Underdetermined System Solver (FOCUSS) [1] is very effective for projection reconstruction MR imaging due to the low-resolution initial estimate from over-sampled k-space center in PR. We applied FOCUSS for projection reconstruction MR imaging using single coils. Extensive experiments with synthetic data as well as brain data confirm that high resolution reconstruction with virtually free of angular aliasing artifacts can be obtained from severely undersampled k-space data.

Theory: FOCUSS is an algorithm to obtain a sparse solution in an underdetermined linear inverse problem such as

$$
y=A x \text {, where } y \in C^{K}, x \in C^{N}, A \text { is } K \times N \text { matrix, and } \mathrm{N}>>\mathrm{K}
$$

Consider the following optimization problem:

find $x=W q$, where $\mathrm{x}$ is known image, $\mathrm{W}$ is a weighting matrix and $\mathrm{q}$ is computed from the constrained minimization problem:

$$
\min \|q\|_{2}^{2} \text {, subject to } \mathrm{AWq}=\mathrm{y}
$$

Them, optimal solution is given by

$$
x=W(A W)^{\dagger} y, \text { where } A^{\dagger}=A^{H}\left(A A^{H}\right)^{-1}
$$

During iteration, the weighting matrix $\mathrm{W}$ can be continuously updated using the previous solution. As the iteration goes on, the FOCUSS is asymptotically equivalent to the $\mathrm{L}_{1}$ minimization problem[1]. Since it is well-known that the $\mathrm{L}_{1}$ minimization problem provides a sparse solution [2], we can expect the FOCUSS solution will be sparse. The convergence of the FOCUSS to a sparse solution was shown rigorously in the original paper [1].

In PR MRI, $y$ is obtained using the inverse Fourier transform of the k-space samples along the radial direction. From the Fourier slice theory, the reconstruction of $x$ can be done using the inverse radon transform. We can formulate the underdetermined linear inverse problem in PR MRI:

$$
\begin{gathered}
y=A x, \text { where } y \in C^{K} \text { is sinogram, } x \in C^{N} \text { is unknown image } \\
A=\int w(x, y) \delta(x \cos \theta+y \sin \theta-s) d x d y
\end{gathered}
$$

In order to reduce the angular sampling without incurring resolution loss, we employ the FOCUSS algorithm. In order to reduce the computational burden, a conjugate gradient method using projection/backprojection operation was developed to implement each step of PR-FOCUSS.

$\underline{\text { Result }}$ : In order to analyze the efficiency of PR-FOCUSS, we apply the PR-FOCUSS algorithm to in vivo brain data. 256 per projections were acquired with an FOV

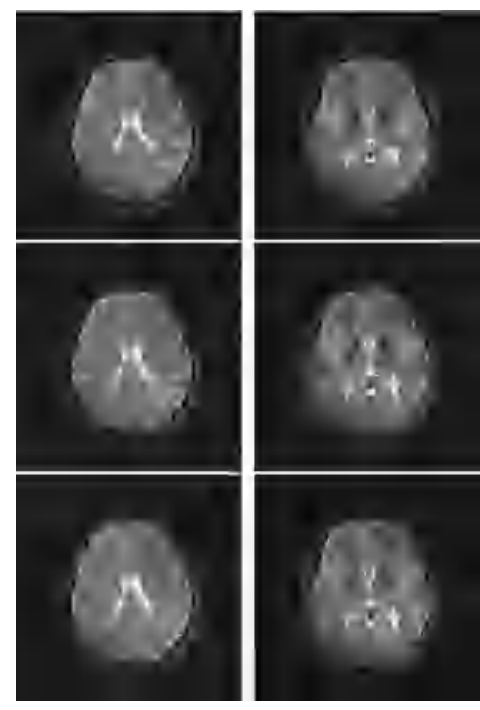

Fig. $11^{\text {st }}$ row: Reconstruction from 190views. $2^{\text {nd }}$ row: Inverse radon images using 51 views. $3^{\text {rd }}$ row: PR-FOCUSS images using 51 views. of $220 \mathrm{~mm}$ x $220 \mathrm{~mm}$ to produce images with 256 x 256 resolution. In this simulation, for fair comparison with standard methods, the reconstruction result from the filtered back-projection is generated. Fig.1 shows the reconstruction results for the 51 views projection data. Aliasing artifacts from the conventional filtered back-projection are totally removed in the reconstruction results by PR-FOCUSS.

Conclusion : We proposed the projection reconstruction FOCUSS (PR-FOCUSS) algorithm to obtain high resolution reconstruction from angularly under-sampled radial k-space data. The FOCUSS algorithm was very effective for the projection reconstruction MRI since the medical images are usually sparse in image domain, and the center area of the under-sampled PR k-space samples still provides a meaningful low resolution image, which is essential for the success of FOCUSS. A conjugate gradient method using projection/back-projection operation was developed to implement each step of the PR-FOCUSS, whose computational burden could be significantly reduced by using the fast projection/back projection algorithms. Simulation results as well as brain data set demonstrated that the proposed PRFOCUSS algorithm is a very effective super-resolution reconstruction algorithm that overcomes the view aliasing artifacts without sacrificing the resolution.

\section{Reference}

[1] I. F. Gorodnitsky and B. D. Rao, "Sparse signal reconstruction from limited data using FOCUSS: re-weighted minimum norm algorithm," IEEE Trans. on Signal Processing, no. 3, pp. 600-616, March 1997.

[2] D.L. Donoho, “Compressed sensing,"IEEE Trans. on Information Theory, vol. 52, no. 4, pp. 1289-1306, April 2006.

Acknoledgement : This work was supported by grant No. 2004-020-12 from the Korea Ministry of Science and Technology (MOST). 